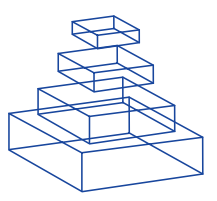

\title{
Neurorobotics: an experimental science of embodiment
}

\author{
Frederic Kaplan \\ Ecole Polytechnique Fédérale de Lausanne, EPFL - CRAFT, Lausanne, Switzerland \\ Correspondence: frederic.kaplan@epfl.ch
}

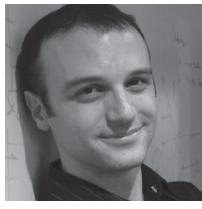

Frederic Kaplan graduated as an engineer of the Ecole Nationale Supérieure des Telecommunications in Paris and obtained a PhD in Artificial Intelligence from the University Paris VI. In his studies and research projects, he has always shown a great interest, both in understanding principles governing living systems and in the construction of biologically inspired systems. He worked 10 years at Sony CSL in Paris. More recently, he joined Ecole Polytechnique Fédérale de Lausanne to supervise a new team of researchers inside the CRAFT laboratory. While exploring novel forms of robotic

devices, he is now continuing collaborations with neuroscientists, psychologists and designers with the objective of creating tighter links between these disciplines and helping the emergence of a novel promising research field: neurorobotics.
At the interface of neuroscience and robotics, neurorobotics is the science and technology of embodied autonomous neural systems. Neural systems include brain-inspired algorithms (e.g. connectionist networks, artificial spiking neural nets), computational models of biological neural networks (e.g. large-scale simulations of neural microcircuits) and actual biological systems (e.g. in vivo and in vitro neural nets). Such neural systems can be embodied in machines with mechanic, pneumatic, electromagnetic or any other forms of physical or virtual actuation. This includes robots, prosthetic, wearable systems, virtual reality environments but at also, at smaller scale, micro-machines and, at the larger scales, furniture and infrastructures.

Neurorobotics is at the confluence of different research trends that emancipated from their original disciplines in the 1980s and 1990s: artificial neural network and computational neuroscience models that are neural systems but not embodied, embodied developmental robotic systems that are embodied and autonomous but not brain-like, and research studies in hybrid biological - artificial systems that are embodied neural systems but with very little autonomy. In each of these fields great progresses have been recently made. Most advanced computing machineries permit now to simulate huge realistic neural network. Promising experiments in epigenetic robotics move from traditional learning of isolated tasks to open-ended developmental trajectories. Pioneering research in hybrid systems permit to investigate symbiotic biological-artificial systems that were previously only in the realm of science fiction. Neurorobotics is the natural meeting point of these converging trends.

The grand challenge of neurorobotics is to build a well-founded experimental science of embodiment. Considered in isolation, neural systems tend to be intrinsically generic, plastic and versatile. However, once embodied and coupled with a given internal or external environment, they become specific and adapted. Neurorobotics offers a novel methodology to understand this process by considering both neural systems and embodiment as experimental variables.

Since the 1950s, robots were essentially seen as fixed bodies on which different programs could be plugged in, like the software and hardware parts of a computer. This dualism has led to a prejudicial divergence between artificial intelligence researchers building 
intelligent programs and roboticists building sophisticated bodies. In the last 1980s, a handful of researchers tried to escape from what appeared to be a technological deadend and pushed forward a reunited view of intelligence, under the name of embodied artificial intelligence or new A.I. They argued that physical bodies and control systems should be intrinsically linked, like two sides of the same coin. The return to the design of complete agents undoubtedly led to some successes, notably for locomotion, sensorimotor learning and navigation in unknown complex environments. However, if such kind of holistic approaches proved to be efficient for designing complex adapted behavior, they were not sufficient to articulate a clear view of developmental processes. For instance, in just a few months, children learn to crawl, stand, walk, jump, hop, run, etc. As they learn these new skills in a continuous incremental manner, their sensorimotor space changes permitting them to investigate novel domains of exploration. This is even clearer with the use of tools or the acquisition of communication skills. This has lead researchers in developmental and epigenetic robotics to present models in which an agent is essentially constituted on the one hand, of a kernel, a set of stable processes that drive developmental dynamics and, on the other hand, of variable body envelopes that change over time. This novel view reverses the classic notion of a fixed body on which different software can be applied to consider a fixed software that can be applied to different kinds of embodiment, potentially changing over time.

Experiments in neurorobotics permit to progress in understanding how the interplay between neural learning dynamics, physical embodiment and environment factors shape developmental trajectories in specific ways, leading, in some circumstances, to openended acquisition of new competences but also, in other cases, to abnormal trajectories. In addition, neurorobotics is expected to provide the technological basis for self-developing devices of a new kind, capable of acquiring new know-how in a continuous and open-ended manner. Understanding how to design such devices and the impact they can have in our daily activities is ofa major importance for the coming years.

Front. Neurosci. (2008) 2, 1:22-23. doi: 10.3389/neuro.01.023.2008

Copyright (C) 2008 Kaplan. This is an open-access publication subject to an exclusive license agreement between the authors and the Frontiers Research Foundation, which permits unrestricted use, distribution, and reproduction in any medium, provided the original authors and source are credited. 